\title{
Improvement of the Surface Properties of Polystyrene Sheets via UV Curable Organic-Inorganic Hybrid Coatings
}

\author{
H. Demirer* ${ }^{*}$ İ. Kartal And M. ÇAKIR \\ Marmara University, Faculty of Technology, 34720 Istanbul, Turkey
}

\begin{abstract}
This study describes the synthesis of silane terminated urethane, aimed at adding into epoxy acrylate, for the coating purposes. For this aim, firstly, silane terminated urethane was synthesized by the reaction between poly(hexamethylene carbonate) diol and 3-isocyanatopropyltrimethoxysilane. Pre-hydrolyzed TEOS and MEMO mixture was added to the silane terminated urethane. This solution was then mixed with organic part (epoxy acrylate and HDDA) to obtain hybrid solutions. Different organic-inorganic hybrid formulations were applied on polystyrene sheet surfaces by using applicators. The performance of the coatings on polystyrene sheets was investigated by applying physical and chemical tests. The results have shown that physical and chemical properties of polystyrene sheet surfaces were significantly improved.
\end{abstract}

DOI: 10.12693/APhysPolA.131.555

PACS/topics: 68.37.-d

\section{Introduction}

During the last two decades, there has been intensive research for the development of functional coatings that exhibit desirable properties, such as wear and chemical resistance, low friction, hydrophobicity, stain resistance etc. Hybrid coatings are considered as the most promising ones among the recent developments in the area of coatings. Hybrid materials reported in the literature are mainly thermally cured. They can also be prepared by using a radiation-curing technique such as using a UV curable resin system [1-4].

UV-curing technique has been widely used in sol-gel processing of hybrid coatings, having UV curable organic functionalities. Hydrolysis and condensation reactions of the inorganic part and UV curable organic part give rise to glassy behavior for the resultant material at room temperature [4-6].

In this study, inorganic containing part (STU+prehydrolyzed TEOS+MEMO) was prepared by the sol-gel method. This solution was then mixed with organic part (epoxy acrylate and HDDA) to obtain hybrid solutions. Different organic-inorganic hybrid formulations were applied on polystyrene sheet surfaces using applicators.

\section{Materials and methods}

\subsection{Materials}

Bisphenol A glycerolate (1 glycerol/phenol) diacrylate (reactive resin), 1,6-hexanediol diacrylate (HDDA, reactive diluent), N,N-dimethylacetamide (DMAc, as a solvent), MEMO (3-methacryloxypropyl trimethoxy silane), Poly (hexamethylene carbonate) diol, 3-isocyanatopropyltrimethoxysilane (ICPTMS), tetraethoxysilane (TEOS), ethanol, and p-toluenesulfonic acid (PTSA, catalyst)

*corresponding author; e-mail: hdemirer@marmara.edu.tr were purchased from Aldrich. Dibutyltin dilaurate (Aldrich) was used as a catalyst. All materials were used as received. Polystyrene sheets of $0.82 \mathrm{~mm}$ thick were purchased from a local supplier. Onium salt (UV initiator) was supplied by Ciba Specialty Chemicals.

\subsection{Synthesis of silane terminated monomer}

Poly (hexamethylene carbonate) diol $(5.01 \mathrm{~g})$ and ICPTMS $(0.515 \mathrm{~g})$ were charged to a three-neck round bottom flask, fitted with a thermometer pocket, water condenser and a magnetic stirrer, filled with $20 \mathrm{wt} . \%$ dimethyl acetamide. Dibutyltin dilaurate, at a concentration of $0.5 \%$, was added to the reaction flask as a catalyst. The temperature was raised to $60^{\circ} \mathrm{C}$ and the mixture was stirred for $24 \mathrm{~h}$. A schematic representation of this reaction is shown in Fig. 1. Completion of the reaction was confirmed by the disappearance of the characteristic $-\mathrm{NCO}$ peak at $2275 \mathrm{~cm}^{-1}$ in the FT-IR spectrum (Fig. 2). The resultant mixture was stored in a refrigerator at $+4{ }^{\circ} \mathrm{C}$ before use. $2.96 \mathrm{~g}$ of pre-hydrolyzed TEOS and MEMO mixture was added to the silane terminated urethane (STU) before mixing with epoxy acrylate and HDDA.

\subsection{Preparation of prehydrolysis of TEOS and hybrid formulations}

Three grams of $(14 \mathrm{mmol})$ TEOS, $0.77 \mathrm{~g}(43 \mathrm{mmol})$ water and p-toluenesulfonic acid (catalyst) in $1.33 \mathrm{~g}$ of ethanol were mixed at room temperature. The water/silicate ratio was calculated as $r=3$. The mixture was left overnight at room temperature. Prepared sol-gel solution (silane terminated urethane (STU)+prehydrolyzed TEOS+MEMO) was mixed with organic part (epoxy acrylate and HDDA) to obtain hybrid solutions. The inorganic part was added drop-by-drop into the stirred epoxy acrylate in a glass tube (open system). Hybrid mixtures were heated to $40^{\circ} \mathrm{C}$ in a vacuum oven for about 10 min to remove the trapped air formed during 


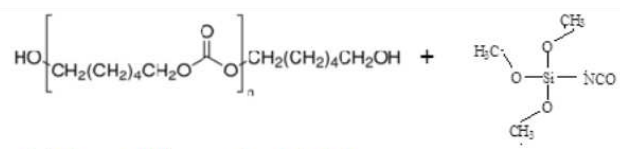

Poly(hexamethylene carbonate) dio Average $M_{n} \sim 2,000$ 3-Isocyanato propyl triethoxy silane (ICPTMS)
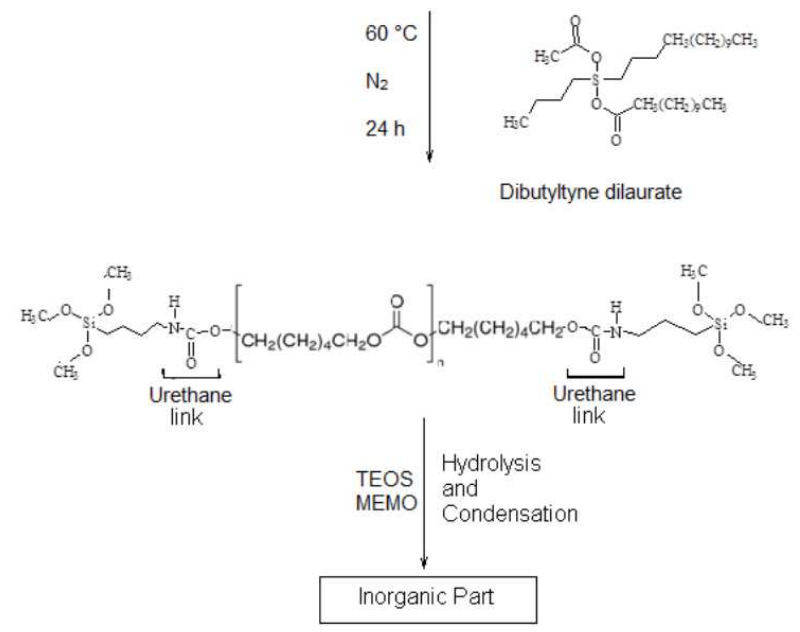

Fig. 1. Flow chart of preparation of sol-gel part.

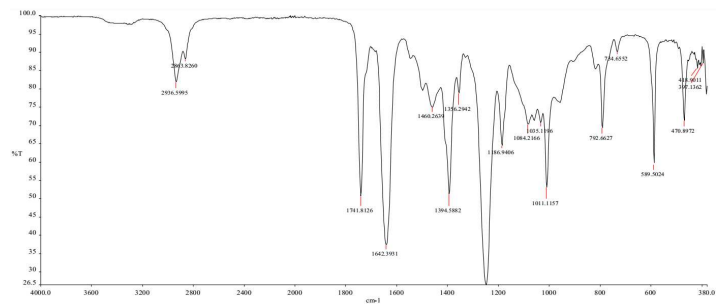

Fig. 2. FT-IR spectrum of silane terminated urethane.

the mixing stage. Finally, $3 \%$ by weight of onium salt was added as a UV initiator. Various hybrid solutions were prepared using different epoxy acrylate/inorganic part solution compositions. There were 6 different compositions in which the inorganic component was varied between $2,4,6,8$ and 10 wt.\%, respectively. The control specimens do not contain inorganic part and were tested to understand the remarkable enhancement in structure properties of the hybrid materials.

\subsection{Preparation of coatings and tensile test specimens}

Each formulation was prepared through mixing until a transparent and homogeneous mixture was obtained in a $25 \mathrm{ml}$ beaker. After air bubbles were eliminated, formulations were homogenously coated upon polystyrene sheets $\left(100 \times 80 \times 0.82 \mathrm{~mm}^{3}\right)$ with a $30 \mu \mathrm{m}$ four-sided applicator, then, hardened in a bench-type UV device (Raycon, $120 \mathrm{~W} / \mathrm{cm}, \lambda_{\max }=365 \mathrm{~nm}$, medium pressure mercury UV lamp). Free films for use as tensile test specimens were also obtained by using these solutions which were poured into the cavities of a Teflon mold and left for curing.

\subsection{Characterization}

In order to characterize the coating performance, various tests were performed on the epoxy acrylate-based hybrids. The FT-IR spectrum of silane terminated urethane was recorded on Shimadzu 8300 FT-IR spectrophotometer. Mechanical properties of hybrid specimens were determined by standard tensile test (ISO 527) to measure the modulus, tensile strength and elongation at break. Tensile experiments were performed on a materials testing system Zwick Z010, using a crosshead speed of $5 \mathrm{~mm} / \mathrm{min}$. The fractured surfaces of the specimens were observed by the JEOL JSM-5910 LV at an acceleration voltage of $20 \mathrm{kV}$. Coating properties were measured in accordance with the corresponding standard test methods as indicated. Cross cut (DIN 53151), tape adhesion (ASTM D-3359), pencil hardness (ASTM D-3363), gloss (ASTM D-5380), stain-resistance (ASTM C-1378) and MEK rub tests (ASTM D-5402) were performed on the coated surfaces. Contact angle measurements were performed at $25^{\circ} \mathrm{C}$ using a Krüss DSA-2 goniometer, interfaced with image-capture software, by injecting a $3 \mathrm{ml}$ liquid drop.

\section{Results}

In this study, inorganic containing part was prepared by the sol-gel method. This solution was then mixed with organic part to obtain hybrid solutions. After reaction, the formation of hydroxyl groups was confirmed by a broad absorption band at $2936 \mathrm{~cm}^{-1}$. The stretching vibration of $\mathrm{SiCH}_{2}$ at $1186 \mathrm{~cm}^{-1}$, CO stretching at $1741 \mathrm{~cm}^{-1}$, the stretching vibration of $\mathrm{SiOCH}_{3}$ at $1084 \mathrm{~cm}^{-1}$ and the stretching vibration of $\mathrm{SiCH}_{2}$ at $792 \mathrm{~cm}^{-1}$ can be seen. Completion of the reaction was confirmed by the disappearance of the characteristic -NCO peak at $2275 \mathrm{~cm}^{-1}$ (Fig. 2).

A total of six different organic-inorganic hybrid formulations were applied on sheet surfaces by using foursided applicators. All physical test results of hybrid coatings are presented in Table I. The pencil hardness test shows that all coatings had at least $6 \mathrm{H}$ pencil hardness value. This value represents the highest measurable value in pencil hardness test. It is believed that the hardness of the all coatings is much above the $6 \mathrm{H}$ pencil hardness.

TABLE I

Physical properties of the hybrid coatings.

\begin{tabular}{c|c|c|c|c|c}
\hline \hline $\begin{array}{c}\text { Inorganic } \\
\text { content } \\
\text { [wt.\%] }\end{array}$ & $\begin{array}{c}\text { Pencil } \\
\text { hardness }\end{array}$ & $\begin{array}{c}\text { Contact } \\
\text { angle } \\
\text { (water) }\end{array}$ & $\begin{array}{c}\text { Cross } \\
\text { cut }\end{array}$ & MEK rub & $\begin{array}{c}\text { Gloss } \\
60^{\circ} \mathrm{C}\end{array}$ \\
\hline 0 & $6 \mathrm{H}$ & 67 & 5 & $>500$ & 174 \\
2 & $6 \mathrm{H}$ & 72 & 2 & $>500$ & 172 \\
4 & $6 \mathrm{H}$ & 77 & 2 & $>500$ & 172 \\
6 & $6 \mathrm{H}$ & 87 & 1 & $>500$ & 175 \\
8 & $6 \mathrm{H}$ & 75 & 0 & $>500$ & 178 \\
10 & $6 \mathrm{H}$ & 73 & 0 & $>500$ & 181
\end{tabular}

It had been proven by many researchers that the increases in the silane content gives rise to an increase of the 
contact angles [6]. Up to $8 \%$ of inorganic content in the hybrid, the contact angle values have been increasing significantly. Above $8 \%$ of inorganic content, however, the decrease of the contact angle values was observed. When the content of sol-gel part is above $8 \%$, silane groups cause the formation of oversized particles, leading to the formation of rounded type surface morphology instead of needle type surface morphology on the coating surfaces. Rounded-type of morphology causes a reduction in contact angle values.

Cross-cut tests have revealed that more than $70 \%$ of the coating had detached from the surfaces in the case of control specimen, i.e. only organic coating. In the coatings containing $2 \%, 4 \%$ and $6 \%$ of inorganic part, there were partial detachments and very low losses. On the other hand, in the coatings containing $8 \%$ and $10 \%$ of inorganic part, excellent adhesion properties were observed. In the present study it was observed that the level of adhesion increased with increasing silane group content. Methyl ethyl ketone (MEK) rub test was performed on all coatings and no change was observed on the coating surfaces, although more than 500 come-and-go scrub cycles were applied. The gloss tests were performed by using a glossmeter at $60^{\circ} \mathrm{C}$. The brightness of coating had also shown an increase, though in a small amount, with increasing silane content.

The stain resistance tests were conducted on the hybrid coatings by using different contamination agents and the results are given in Table II. Contamination agents were applied onto the coated surfaces, cleaned with water after a defined contact time, and changes in coating surfaces were investigated. All coated surfaces have shown excellent stain resistance against contamination agents. This shows the effectiveness of the formulations with and without the inorganic part.

TABLE II

The stain resistance of the hybrid coatings.

\begin{tabular}{c|c|c|c|c|c}
\hline \hline \multirow{2}{*}{$\begin{array}{c}\text { Inorganic } \\
\text { content, } \\
\text { [wt.\%] }\end{array}$} & \begin{tabular}{c} 
Stain resistance test agent/Contact time \\
\cline { 2 - 6 }
\end{tabular} & $\begin{array}{c}\text { Coffee } \\
16 \mathrm{~h}\end{array}$ & $\begin{array}{c}\text { Acetic Acid, } \\
30 \% \\
10 \mathrm{~min}\end{array}$ & $\begin{array}{c}\mathrm{NaOH}, \\
25 \% \\
10 \mathrm{~min}\end{array}$ & $\begin{array}{c}\mathrm{H}_{2} \mathrm{O}_{2}, \\
20 \% \\
10 \mathrm{~min}\end{array}$ \\
\hline 0 & 5 & 5 & 5 & 5 & 5 \\
2 & 5 & 5 & 5 & 5 & 5 \\
4 & 5 & 5 & 5 & 5 & 5 \\
6 & 5 & 5 & 5 & 5 & 5 \\
8 & 5 & 5 & 5 & 5 & 5 \\
10 & 5 & 5 & 5 & 5 & 5 \\
\hline
\end{tabular}

The stain-resistance evaluation: 1: heavy staining, 2: considerable staining, 3: noticeable staining, 4: slight staining, 5: no staining

The contact angle is conventionally measured through the liquid, where a liquid interface meets a solid surface. If the contact angle is greater than $90^{\circ}$, the surface exhibits hydrophobic properties [7]. Contact angle values of the hybrid coatings are given in Table I. Contact angle values had increased with the increase of inorganic content of coating formulation up to $8 \%$. Then, despite the increase in the content of inorganic part, the value of the contact angle had decreased gradually. The decline of the contact angle values was considered to be due to agglomerated silica in the structure. In Fig. 3 contact angle images for water on plated surfaces are presented.

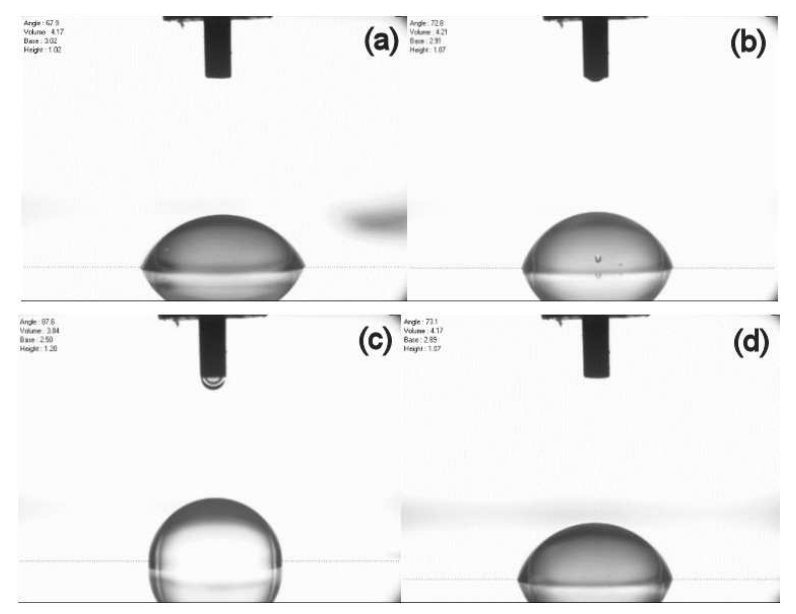

Fig. 3. Contact angle images of hybrid coatings (a) $0 \%$, (b) $2 \%$, (c) $6 \%$, (d) $10 \%$.

Tensile and Izod impact properties of hybrid specimens are given in Table III. Tensile strength and percentage of elongation decrease with the increase in inorganic content. With the increasing sol-gel content, the amount of inorganic part increases within the organic structure. Increasing inorganic content within the hybrid material gives rise to the reduction in tensile strength and elongation to break. In this study with the increasing inorganic content a reduction in tensile strength and elongation to break were observed [8].

TABLE III

Tensile and Izod impact properties of hybrid specimens.

\begin{tabular}{c|c|c|c}
\hline $\begin{array}{c}\text { Inorganic } \\
\text { content } \\
\text { [wt.\%] }\end{array}$ & $\begin{array}{c}\text { Tensile } \\
\text { strength } \\
{[\mathrm{MPa}]}\end{array}$ & $\begin{array}{c}\text { Strain }(\varepsilon) \\
{[\%]}\end{array}$ & $\begin{array}{c}\text { Izod impact } \\
\text { strength }\end{array}$ \\
\hline 0 & 20.48 & 3.85 & 5.0 \\
2 & 18.78 & 3.55 & 5.4 \\
4 & 16.92 & 3.55 & 6.2 \\
6 & 14.75 & 3.43 & 7.1 \\
8 & 13.65 & 3.44 & 8.0 \\
10 & 11.20 & 3.21 & 9.2
\end{tabular}

As can be seen from the Table III, with the addition of silane terminated urethane to epoxy resin, the impact strength of resultant hybrids had increased considerably. Impact strength of $10 \%$ silane terminated urethane added epoxy acrylate had increased from 5 to $9.2 \mathrm{~kJ} / \mathrm{m}^{2}$. This increase in the impact strength was attributed to the presence of urethane, which caused an increase in 
the toughness of epoxyacrylate matrix [9]. The morphology of hybrid free films were examined by SEM. Figure 4 shows the fracture surface of a specimen having $6 \%$ of inorganic content. Figure 4 shows the presence of fracture bands. These fracture bands reveal that the fracture had occurred through the partial plastic deformation, which is the source of energy absorption during fracture [10].

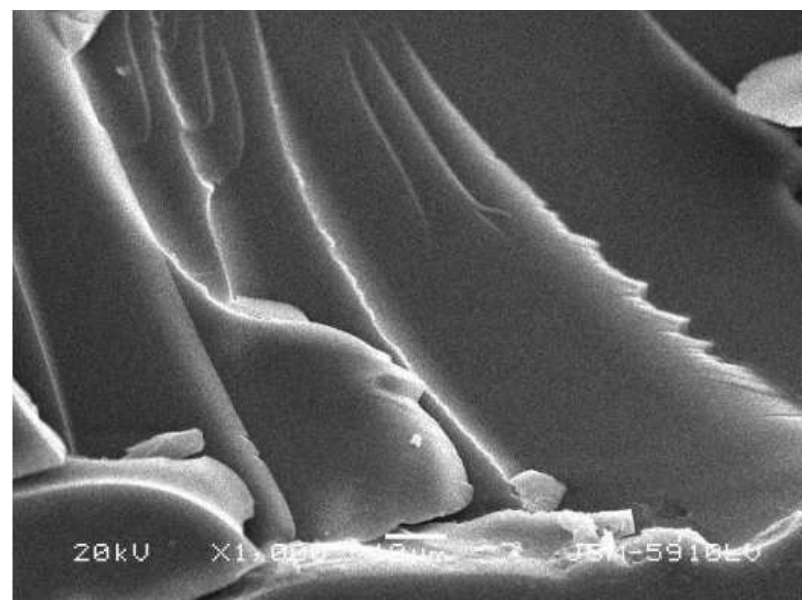

Fig. 4. SEM images of hybrid coating having $6 \%$ of inorganic content.

\section{Conclusions}

With the help of a continuous UV radiation equipment, a well and deeply crosslinked coating layer was obtained on the polystyrene sheet surface. From the results it was found that physical properties of the polystyrene could substantially be improved by the application of hybrid coatings. Although a polystyrene sheet surface can easily be scratched by a finger nail, in its as received condition, the hardness of the surface had reached the $6 \mathrm{H}$ pencil hardness after coating. In MEK rub test, at the end of 500 cycles there was no change on the surface of the hybrid coated polystyrene, which is normally not resistant to MEK. In brightness measurements there was no meaningful increase in the value of brightness, since polystyrene sheet has a highly bright surface in its original state. Stain resistance properties of the coated plates were found to be excellent. Regarding the contact angle measurements, the coating did not appear to have complete hydrophobicity. Thus, it can be said that the coatings have stain-resistance but have no self-cleaning feature. The bond between the coating material and the polystyrene surface was found to be good enough. Polystyrene sheet was made scratch-resistant through hybrid coating, without compromising the brightness. Thus, a solvent-resistant coating that can easily be used in outdoor environments and cleaned with cleaning materials available, without scratches and dissolving has been developed.

\section{References}

[1] J. Maul, B.G. Frushour, J.R. Kontoff, H. Eichenauer, K-H. Ott, C. Schade, Polystyrene and Styrene Copolymers in: Ullmann's Encyclopedia of Industrial Chemistry, Wiley-VCH, 2007.

[2] M. Cakir, I. Kartal, Z. Yildiz, Text. Res. J. 84, 1528 (2014).

[3] M. V. Kahraman, Z.S. Akdemir, I. Kartal, N. Kayaman-Apohan, A. Gungor, Polym. Adv. Technol. 22, 981 (2009).

[4] R.D. van de Grampel, W. Ming, W.J.H. van Gennip, F. van der Velden, J. Laven, J.W. Niemantsverdriet, R. van der Linde, Polymer 46, 10531 (2005).

[5] Z. Zong, J. He, M.D. Soucek, Progress Organic Coat. 53, 83 (2005).

[6] V. Kumar, N. Misra, J. Paul, Y.K. Bhardwaj, N.K. Goel, S. Francis, K.S.S. Sarma, L. Varshney, Progress Organic Coat. 76, 1119 (2013).

[7] J. Gao, X. Wang, Y. Wei, W. Yang, J. Fluor. Chem. 127, 282 (2006)

[8] M. Cakir, I. Kartal, H. Demirer, R. Samur, Sci. Res. Essays 7, 805 (2012).

[9] İ. Kartal, Asian J. Chem. 26, 6018 (2014).

[10] H. Beytut M. Çakır, İ. Kartal, M. Sengül, D. Tutak, Asian J. Chem. 27, 3854 (2015). 